\title{
Connectivity pattern differences bilaterally in the cerebellum posterior lobe in healthy subjects after normal sleep and sleep deprivation: a resting-state functional MRI study
}

\author{
This article was published in the following Dove Press journal: \\ Neuropsychiatric Disease and Treatment \\ 26 May 2015 \\ Number of times this article has been viewed
}

\author{
Xuming Liu' \\ Zhihan Yan ${ }^{2}$ \\ Tingyu Wang' \\ Xiaokai Yang' \\ Feng Feng ${ }^{3}$ \\ Luping Fan' \\ Jian Jiang ${ }^{4}$ \\ 'Department of Radiology, The \\ Third Clinical Institute Affiliated \\ to Wenzhou Medical University, \\ Wenzhou, ${ }^{2}$ Department of Radiology, \\ The 2nd Affiliated Hospital of \\ Wenzhou Medical University, \\ Wenzhou, ${ }^{3}$ Peking Union Hospital, \\ Peking Union Medical College, \\ Chinese Academy of Medical Sciences, \\ Beijing, ${ }^{4}$ Department of Radiology, The \\ First Affiliated Hospital of Nanchang \\ University, Nanchang, People's \\ Republic of China
}

Correspondence: Xuming Liu Department of Radiology, The Third Clinical Institute Affiliated to Wenzhou Medical University, Number 57 , CangHouStreet, LuCheng District, Wenzhou 325000, Zhejiang, People's Republic of China

Tel +8657788059863

Email liuxumingdo@I26.com

Jian Jiang

Department of Radiology, The First Affiliated Hospital of Nanchang University, Number 17, YongWai Zheng Street, DongHu District, Nanchang 330006, Jiangxi, People's Republic of China

Tel +8679188692582

Email jij2002cn@yahoo.com.cn
Objective: The aim of this study was to use functional magnetic resonance imaging (fMRI) technique to explore the resting-state functional connectivity (rsFC) differences of the bilaterial cerebellum posterior lobe (CPL) after normal sleep (NS) and after sleep deprivation (SD).

Methods: A total of 16 healthy subjects (eight males, eight females) underwent an fMRI scan twice at random: once following NS and the other following 24 hours' SD, with an interval of 1 month between the two scans. The fMRI scanning included resting state and acupuncture stimulation. The special activated regions located during the acupuncture stimulation were selected as regions of interest for rsFC analysis.

Results: Bilateral CPLs were positively activated by acupuncture stimulation. In the NS group, the left CPL showed rsFC with the bilateral CPL, bilateral frontal lobe (BFL), left precuneus and right inferior parietal lobule, while the right CPL showed rsFC with the bilateral temporal lobe, right cerebellum anterior lobe, right CPL, left frontal lobe, left anterior cingulate, right posterior cingulate, and bilateral inferior parietal lobule. In the SD group, the left CPL showed rsFC with the left posterior cingulate gyrus bilateral CPL, left precuneus, left precentral gyrus, BFL, and the left parietal lobe, while the right CPL showed rsFC with bilateral cerebellum anterior lobe, bilateral CPL, left frontal lobe and left temporal lobe. Compared with the NS group, the left CPL had increased rsFC in the SD group with the right inferior frontal gyrus, right fusiform gyrus, right cingulate gyrus, right thalamus, and bilateral precuneus, and decreased rsFC with the BFL, while the right CPL had increased rsFC with the left superior frontal gyrus and decreased rsFC with the left precentral gyrus, right superior temporal gyrus, and the BFL.

Conclusion: Bilateral CPL are possibly involved in acupuncture stimulation in different manners, and the right CPL showed more rsFC impairment.

Keywords: fMRI, functional connectivity, acupuncture, resting-state functional magnetic resonance imaging

\section{Introduction}

We each nearly spend a third time of our life asleep; sleep is a necessary physical need in human life. Sleep deprivation (SD), widespread in today's society and associated with a number of clinical conditions, has a detrimental effect on attention, working memory, executive functioning, and emotion. ${ }^{1-4}$ Short-term SD increases the morbidity of daytime sleepiness and has been shown to have a negative impact on metabolic 
parameters such as glucose tolerance and insulin sensitivity, and places individuals at a greater risk of serious disease, such as diabetes. Long-term SD is harmful to health and can lead to multisystem and multiorgan dysfunction, causing maladaptive emotional regulation, exaggerated neural reactivity, aversive experiences, and negative metabolic, psychological, physiological, or even behavioral reactivity. ${ }^{5-8}$ However, their mechanisms remain unclear.

In recent years, there has been a serious revolution in understanding of cerebellar function. The cerebellum was considered to be involved exclusively in motor coordination, but new findings suggest that the cerebellum plays a role in multiple functional domains. ${ }^{9-14}$ The notion that the cerebellum is involved in emotional processing has recently gained popularity, and there is a large body of empirical research in patients with cerebellar damage that has demonstrated the region is related to emotional regulation. ${ }^{14,15}$ Furthermore, anatomical associations also suggest a possible mechanism through which the cerebellum can affect mood processing. These findings help to broaden our view of cerebellar function. This is a crucial implication in light of mounting evidence for cerebellar involvement in various neurologic and psychiatric conditions, including primary insomnia, ${ }^{16}$ autism, ${ }^{17}$ depression, ${ }^{18}$ mood disorders, ${ }^{19}$ and obstructive sleep apnea. ${ }^{20}$ However, the role of the cerebellum has not yet been paid enough attention.

Sleep disorders can be treated by drug intervention, but while medications have certain curative effects, they may also have negative effects such as drug addiction. $\mathrm{SD}$, as a stressor, can break the functional network and ultimately lead to decline in overall immunity and endocrine disorders. However, acupuncture can correct the imbalance and effectively improve sleep. Acupuncture is a noninvasive, safe, and effective method for the management of insomnia in adolescents, with good compliance and no adverse effects. ${ }^{21}$ A previous functional magnetic resonance imaging (fMRI) study found that acupuncture could play a role in ameliorating brain dysfunction and could noninvasively alter the distribution of resting-state networks of SD subjects. ${ }^{22}$

Different brain regions are recognized for their remarkable functional specificity, and our daily life depends on the ability to rapidly coordinate and integrate the distinct operations of these specific regions. Once one region is dysfunctional, corresponding symptoms will appear. Specific types of information processing, such as acupuncture processing, may be reflected in activation in a single brain region or synchronously across multiple brain regions. Needling a specific acupoint can specifically activate functional activities of specific parts of the cerebral cortex. ${ }^{23,24}$ Some studies found that cerebellum posterior lobe (CPL) involvement in acupuncture information processing was the common neural pathway of different acupoints..$^{25,26}$

It is proposed that resting-state functional magnetic resonance imaging (rfMRI), one of the current areas of interest in neuroimaging and one that is suitable for central mechanism research, can detect the spontaneous neuronal activity of the human brain and provide new insights into the pathophysiology of disease because of its advantages in not requiring exposure to radioactive tracers, accurate positioning, or combination with functional and structural imaging. ${ }^{27}$ The exploration and use of rfMRI data have grown hugely in recent years. ${ }^{28}$

Resting-state functional connectivity ( $\mathrm{rsFC}$ ), one of the important developments in rfMRI, involves calculation of the correlation between a signal time course from one brain region and the time courses from the rest of the brain regions. In recent years, increasing attention has been devoted to exploring interregional connectivity during a specific stimulation, defined as "functional connectivity". ${ }^{29}$ The rsFC indicates temporal correlations between neural or hemodynamic signals arising from distinct brain regions, and it measures the signal synchrony of the amplitude of low-frequency fluctuation activity among different brain areas.

rsFC analysis reflects the complexity of the neural network, with reduced and increased rsFC potentially involving different compensatory ways of multiple pathways. The partial connectivity approach may be analogous to the canonical subtraction method for assessing the specificity of stimulation-evoked activation. In addition, pattern analysis uses resting connectivity to predict the response of brain regions during stimulations. These techniques suggest new ways to link stimulation and resting paradigms. The ability to characterize fine-grained networks using partial connectivity and to link this connectivity to stimulation activation may move us closer to understanding the relationship between the cerebellum and SD.

In the study reported here, we hypothesized that the cerebellum would be specifically activated by acupuncture stimulation and show a specific connectivity pattern. For a more comprehensive understanding of the interaction between the cerebellum and SD, we examined whether the cerebellum was specifically activated by acupuncture stimulation and the statistical differences in $\mathrm{rsFC}$ of the left and right cerebellum between SD and normal sleep (NS). 


\section{Methods}

\section{Subjects}

Sixteen healthy subjects (eight males, eight females), ranging in age from 21 to 25 years old (mean age $=22.1 \pm 0.8$ years), who were university students responded to a web-based questionnaire were recruited for this study. The sleeping habits and sleep quality of all participants were monitored throughout 1 week by a Fitbit Flex tracker (http://help.fitbit.com/) worn by each participant, and only those whose data indicated habitual good sleep were recruited for this study. ${ }^{16}$ All subjects met the following criteria as in previous studies: : $22,25^{2}$ (1) no symptoms associated with sleep disorders and no history of any psychiatric or neurologic disorders; (2) right-handed; (3) a good sleeping habit - that is, $>6.5$ hours of sleep per night, falling asleep no later than $1 \mathrm{am}$, and waking-up no later than $9 \mathrm{am}$; (4) had a good sleep onset and/or maintenance, and no history of swing shift, shift work, or sleep complaints; (5) regular dietary habits, and did not consume any stimulants, alcohol, tea, cigarettes, medications, or caffeine for at least 3 months prior to the study; (6) no foreign implants in the body, and no inborn or other acquired diseases; (7) moderate body shape and weight; (8) a Pittsburgh Sleep Quality Index score $<5$, and Hamilton Depression Rating Scale and Hamilton Anxiety Rating Scale scores $<7$.

\section{Design}

Each of the subjects, in random sequence, underwent the session twice: once was after NS, and the other was after total of 24 hours' SD. SD was conducted in a specialized room and started from $7 \mathrm{pm}$ and ended at $7 \mathrm{pm}$ in the following day. During the SD session, subjects were allowed to engage in leisure activities such as watching TV, surfing the Internet, playing cards, and reading. Vigorous physical activity prior to the scans was not permitted.

Each subject underwent brain fMRI scanning during resting state and during acupuncture stimulation. Acupuncture was performed with a block design; that is, a "restmanipulating needle-rest-manipulating needle" program. To avoid the effect of the acupuncture on the brain activity, the interval between the two scans was 1 month.

All volunteers were informed of the purposes, methods, and potential risks of the study. Before the fMRI session, they were asked to go to the toilet (emptying feces and urine) and rest quietly for 30 minutes. The subjects were advised they should keep their head still to avoid audio stimulus and their eyes open during the fMRI scans. De-qi assessment was undertaken in all subjects after the scans (see "Assessment of de-qi" subsection). The study was approved by the Human Research Ethics Committee of our department.
All subjects participated voluntarily and gave their written informed consent.

\section{Acupuncture treatment}

Acupuncture was performed by the same acupuncturist on all participants. Manual acupuncture stimulation was performed at the acupoint SP6 on the right shank (Sanyinjiao, located in the medial lower shank, $9-10 \mathrm{~cm}$ proximal to the upper border of the medial malleolus [ankle bone] between the posterior border and facies medialis of the tibia). The fine silver needles used in the acupuncture protocol were sterile and disposable, and would not distort the magnetic resonance (MR) images.

To assess the specific effects triggered by acupuncture stimulation, the mentioned fine silver needle, measuring $0.3 \mathrm{~mm}$ in diameter and $40.0 \mathrm{~mm}$ in length, was quickly inserted obliquely into the shank at the right of the SP6 acupoint at a depth of approximately $15 \pm 2 \mathrm{~mm}$. After cleaning the skin with alcohol and marking the acupoint, the needle was then rotated slowly at a frequency of 60 times per minute and rotation range of $\pm 180^{\circ}$ when the acupuncturist felt de-qi. The details are presented in a previous study. ${ }^{25}$

\section{Assessment of de-qi}

De-qi of acupuncture plays a key role in treatment. "De-qi", a subjective feeling of perceived stimulation felt by subjects, is used as evidence to judge the selection of the exact acupoint, curative effect, and prognosis. The intension of de-qi was assessed by subjects' subjective description rather than objective medical instruments. Subjects were asked to rate each component of the de-qi feeling during the stimulation at the end of the session using a visual analog scale, which included soreness, numbness, fullness, heaviness, and dull pain.

In the study, the feeling project was divided into six grades: insentience (a score of $0-1$ ), mild feeling (2-3), moderate feeling (4-5), generally strong feeling (6-7), extremely strong feeling (8-9), and hard to bear (10). Subjects who scored items $\leq 1$ and/or $\geq 8$ would be excluded.

\section{Magnetic resonance imaging parameters}

Magnetic resonance imaging (MRI) scanning was performed on a 3-Tesla MR scanner (Trio, Siemens, Erlangen, Germany). The scan consisted of located images, T1 sagittal images, and functional images. The parameters of functional imaging between resting state and stimulation state were the same. High-resolution T1-weighted images were acquired with a three-dimensional spoiled gradient-recalled sequence in a sagittal orientation: 176 images (repetition time $=1,900 \mathrm{~ms}$, cho time $=2.26 \mathrm{~ms}$, thickness $=1.0 \mathrm{~mm}$, gap $=0 \mathrm{~mm}$, 
matrix $=256 \times 256$, field of view $=240 \times 240 \mathrm{~mm}$, flip angle $=15^{\circ}$ ) were obtained. Functional images (repetition time $=3,000 \mathrm{~ms}$, echo time $=30 \mathrm{~ms}$, thickness $=5 \mathrm{~mm}$, gap $=1 \mathrm{~mm}$, matrix $=64 \times 64$, flip angle $=90^{\circ}$, field of view $=240 \times 240 \mathrm{~mm}$, 36 axial slices with gradient-recalled echo-planar imaging pulse sequence) covering the whole brain were obtained.

\section{Data analysis \\ Data preprocessing}

Functional data were checked by MRIcro software (www. MRIcro.com) to exclude defective data. The first ten time points of the functional images were discarded due to the possible instability of the initial MRI signal and the participants' adaptation to the scanning environment. On the basis of MATLAB2010a (Mathworks, Natick, MA, USA), the rest of the data preprocessing was performed by Data Processing Assistant for Resting-State fMRI software (v 2.3; http:// rfmri.org/DPARSF), including Digital Imaging and Communications in Medicine standards for form transformation, slice timing, head-motion correction, spatial normalization, smooth with a Gaussian kernel of $6 \times 6 \times 6 \mathrm{~mm}^{3}$ full-width at half-maximum. Motion time courses were obtained by estimating the values for translation ( $\mathrm{mm}$ ) and rotation (degrees) for each subject. The participants who had more than 1.5 $\mathrm{mm}$ maximum displacement in $\mathrm{x}, \mathrm{y}$, or $\mathrm{z}$ and $1.5^{\circ}$ of angular motion in all rfMRI scans would be rejected. The Friston six head-motion parameters were used to regress out headmotion effects based on recent work showing that higherorder models were more effective in removing head-motion effects. ${ }^{30,31}$ Linear regression was also applied to remove other sources of spurious covariates along with their temporal derivatives, including the signal from ventricular regions of interest (ROIs) and the signal from a region centered in the white matter. ${ }^{32}$ Of note, the global signal was not regressed out in the present data, as described by previous studies, ${ }^{18,30,31}$ for the reason that it is still controversial to remove the global signal in the preprocessing of resting-state data. ${ }^{16,29,32,33}$ After head-motion correction, the fMRI images were spatially normalized to the Montreal Neurological Institute space using the standard echo-planar imaging template and resampling the images at a resolution of $3 \times 3 \times 3 \mathrm{~mm}$.

\section{Acupuncture stimulation data}

To investigate the acupuncture effect, the preprocessing data were analyzed by Statistical Parametric Mapping (SPM; v 5) software, and a general linear model was used to analyze the block-designed data. The whole activated figure of each subject was acquired. One-sample $t$-test results were presented by Xjview and Resting-State fMRI Data Analysis Toolkit (REST, v 1.8). The statistical map for active brain regions was set at a cluster extent of $\geq 13$ and a voxel level of $P<0.001$, which was corrected for multiple comparison by AlphaSim.

rsFC

After preprocessing, temporal filtering settings were applied using a band-pass filter $(0.01-0.08 \mathrm{~Hz})$ to reduce lowfrequency drift and physiological high frequency respiratory and cardiac noise. The influence of covariant (including headmotion parameters, global mean signal, white matter signal, and cerebrospinal fluid signal) should be eliminated. The average time series from the ROIs were extracted from the residual image. To make the data fit the normal distribution, we calculated the Pearson's correlation coefficient between the ROIs and other voxels of the whole brain. The coefficient was subjected to Fisher's Z transformation, with the Z-value representing the function connection coefficient. The different brain regions from the stimulation were selected as ROIs for $\mathrm{rsFC}$ analysis. To reduce the global effects of variability across the participants, the rsFC of each voxel was divided by the global mean value for each participant. The details are presented in a previous study. ${ }^{27}$

\section{Behavioral analysis}

To examine changes in behavior performance over the course of the SD sessions, the mean reaction time (RT) and false rates (button pressed following a cue) about the accuracy of performance were computed. ${ }^{34}$

\section{Statistics}

The behavioral deficits were evaluated using two-pair $t$-tests using IBM SPSS (v 21.0) software, and the statistical threshold was set at $P<0.05$.

The one-sample $t$-tests and two-pair $t$-tests results were determined with SPM (v 8; http://www.fil.ion.ucl.ac.uk/ $\underline{\mathrm{spm}}$ ), with age and years of education as nuisance covariates of no interest. A significance level of $P<0.001$ for individual voxels and a continuous voxel size $\geq 13$ were used to determine statistical significance, which was corrected for multiple comparison by AlphaSim with a cluster threshold of $P<0.05$. The results were presented by xjView and REST.

\section{Results}

\section{De-qi and head-motion}

No significant structure abnormalities were found in any subject using plain MR scanning. One subject who had more than $1.5 \mathrm{~mm}$ maximum displacement in $\mathrm{x}, \mathrm{y}$, or $\mathrm{z}$ and $1^{\circ}$ of 
Table I Altered resting-state functional connectivity areas of the left cerebellum posterior lobe after normal sleep

\begin{tabular}{|c|c|c|c|c|c|}
\hline \multirow[t]{2}{*}{ Brain } & \multirow[t]{2}{*}{$R / L$} & \multirow[t]{2}{*}{ Voxels } & \multicolumn{3}{|c|}{ MNI } \\
\hline & & & $\mathbf{x}$ & $\mathbf{Y}$ & $\mathbf{Z}$ \\
\hline \multicolumn{6}{|l|}{ Positive correlation } \\
\hline Cerebellum posterior lobe & L & 315 & -48 & -66 & -33 \\
\hline Cerebellum posterior lobe & $\mathrm{R}$ & 130 & 42 & -54 & -42 \\
\hline Cerebellum posterior lobe & $\mathrm{R}$ & 49 & 30 & -81 & -30 \\
\hline Cerebellum posterior lobe & L & 34 & -30 & -87 & -27 \\
\hline Superior frontal gyrus & $\mathrm{R}$ & 13 & 33 & 57 & 0 \\
\hline Superior frontal gyrus, middle frontal gyrus & L & 27 & -24 & 60 & 6 \\
\hline Superior frontal gyrus, middle frontal gyrus & $\mathrm{R}$ & 22 & 27 & 57 & 33 \\
\hline Superior frontal gyrus & L & 18 & -27 & 48 & 30 \\
\hline \multicolumn{6}{|l|}{ Negative correlation } \\
\hline Precuneus & L & 41 & -18 & -75 & 36 \\
\hline Inferior parietal lobule & $\mathrm{R}$ & 25 & 36 & -48 & 54 \\
\hline
\end{tabular}

Note: A significance level of $P<0.00 \mathrm{I}$ for individual voxels and a continuous voxel size $\geq 13$ were used to determine statistical significance, corrected for AlphaSim. Abbreviations: R, right; L, left; MNI, Montreal Neurological Institute.

angular motion, and one subject whose score was greater than eight in the assessment of de-qi, were excluded from this study.

\section{Performance findings}

There was no significant difference in accuracy between the SD and NS groups. The SD group had significantly longer RT than the NS group (NS =2,010.40 \pm 227.17 [ms], $\mathrm{SD}=2,275.10 \pm 176.66[\mathrm{~ms}], t=-3.858$, degrees of freedom $[d f]=15, P<0.002)$.

\section{Acupuncture stimulation and ROI definition}

Since there are no accepted coordinates in the cerebellum, we predefined these from a statistical map by selecting different areas. Fortunately, a previous study found that both left and right CPL areas were positively activated by acupuncture stimulation both in a NS group and in a SD group. ${ }^{35}$ Thus, both sides of CPLs were selected as ROIs for future rsFC in this study.

\section{rsFC results}

\section{Altered rsFC regions in the NS group}

In the NS group, the left CPL showed positive rsFC with both sides of the CPL, both sides of the superior frontal gyrus, and both sides of the middle frontal gyrus, and showed negative rsFC with the left precuneus ( $\mathrm{PrC})$ and right inferior parietal lobule (IPL; Table 1, Figure 1). Further, the right CPL showed positive rsFC with the left cluster of the middle temporal gyrus and inferior temporal gyrus, right cerebellum

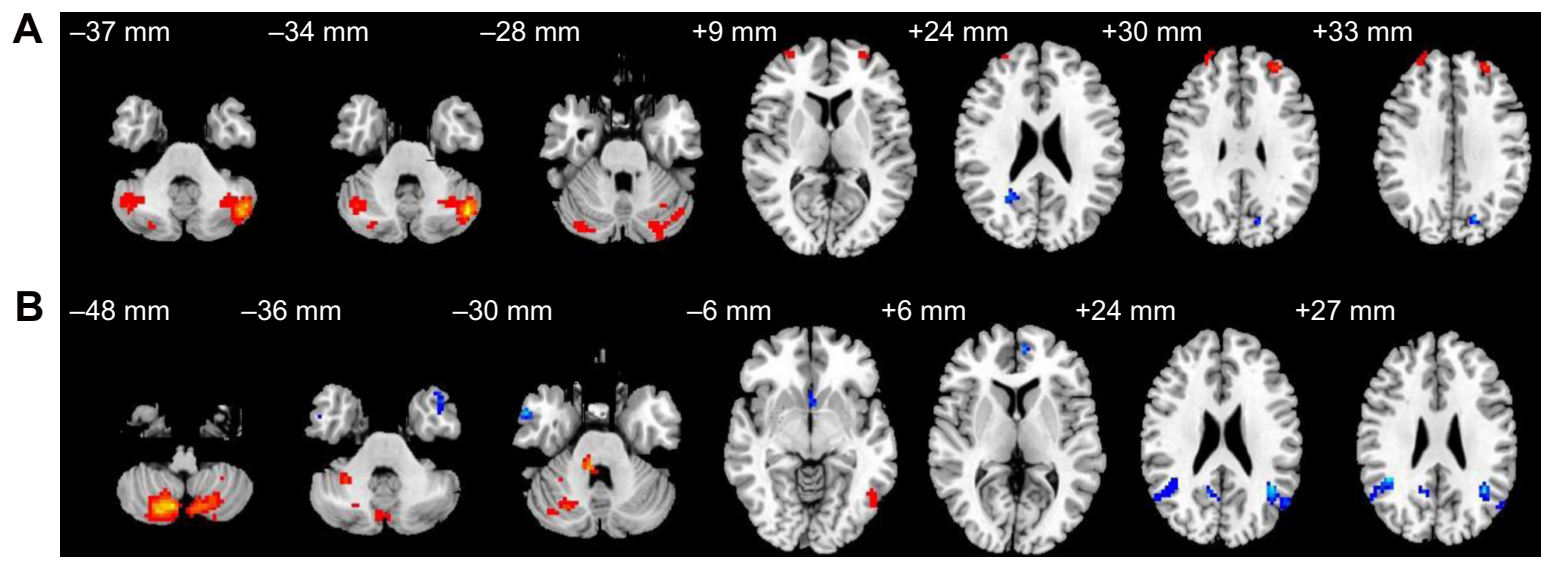

Figure I Altered resting-state functional connectivity $(\mathrm{rsFC})$ areas of the cerebellum posterior lobe $(\mathrm{CPL})$ in the normal sleep group.

Notes: The rsFC areas of the left CPL were seen in the left precuneus, right inferior parietal lobule, bilaterally in the CPL, bilaterally in the superior frontal gyrus, and bilaterally in the middle frontal gyrus (A), while the rsFC areas of the right CPL were seen in the left middle frontal gyrus, left anterior cingulate, left angular gyrus, right posterior cingulate, right inferior parietal lobule, right cerebellum anterior lobe, right CPL, bilaterally in the superior frontal gyrus, bilaterally in the middle temporal gyrus, bilaterally in the inferior temporal gyrus, and supramarginal gyrus (B). 
Table 2 Altered resting-state functional connectivity areas of the right cerebellum posterior lobe after normal sleep

\begin{tabular}{|c|c|c|c|c|c|}
\hline \multirow[t]{2}{*}{ Brain } & \multirow[t]{2}{*}{$R / L$} & \multirow[t]{2}{*}{ Voxels } & \multicolumn{3}{|c|}{ MNI } \\
\hline & & & $\mathbf{x}$ & $\mathbf{Y}$ & $\mathbf{Z}$ \\
\hline \multicolumn{6}{|l|}{ Positive correlation } \\
\hline Cerebellum posterior lobe & $\mathrm{R}$ & 759 & 15 & -75 & -45 \\
\hline Cerebellum anterior lobe, cerebellum posterior lobe & R & 41 & 30 & -48 & -33 \\
\hline Cerebellum anterior lobe & $\mathrm{R}$ & 17 & 9 & -39 & -30 \\
\hline Middle temporal gyrus, inferior temporal gyrus & $\mathrm{L}$ & 21 & -51 & -66 & -6 \\
\hline \multicolumn{6}{|l|}{ Negative correlation } \\
\hline Superior temporal gyrus & L & 15 & -45 & 12 & -36 \\
\hline Middle temporal gyrus, inferior temporal gyrus & $\mathrm{R}$ & 18 & 60 & 3 & -27 \\
\hline Middle frontal gyrus & L & 15 & -6 & 54 & 6 \\
\hline Posterior cingulate & $\mathrm{R}$ & 37 & 6 & -57 & 18 \\
\hline Superior temporal gyrus, middle temporal gyrus, angular gyrus & L & 54 & -57 & -60 & 18 \\
\hline Inferior parietal lobule, superior temporal gyrus, middle temporal gyrus, supramarginal gyrus & $\mathrm{R}$ & 100 & 42 & -48 & 27 \\
\hline Superior temporal gyrus, middle temporal gyrus, supramarginal gyrus & L & 37 & -39 & -57 & 27 \\
\hline Anterior cingulate & $\mathrm{L}$ & 18 & -3 & 6 & 9 \\
\hline
\end{tabular}

Note: A significance level of $P<0.001$ for individual voxels and a continuous voxel size $\geq 13$ were used to determine statistical significance, corrected for AlphaSim. Abbreviations: R, right; L, left; MNI, Montreal Neurological Institute.

anterior lobe and right CPL, and negative rsFC with the left middle frontal gyrus, left anterior cingulate, right inferior temporal gyrus, right posterior cingulate, and both sides of the IPL, both sides of the superior temporal gyrus, and both sides of the middle temporal gyrus (Table 2, Figure 1).

\section{Altered rsFC regions in the SD group}

In the SD group, left CPL showed positive rsFC with the left posterior cingulate gyrus and both sides of the CPL, and showed negative rsFC with the left $\mathrm{PrC}$, left precentral gyrus, left inferior frontal gyrus, left superior parietal lobule, left postcentral gyrus, left IPL, and right middle frontal gyrus (Table 3, Figure 2). Moreover, the right CPL showed positive rsFC with both sides of the cerebellum anterior lobe and both sides of the CPL, and showed negative rsFC with the left cluster of the middle frontal gyrus, paracentral lobule, and superior temporal gyrus, and left cluster of the superior temporal gyrus and middle temporal gyrus (Table 4, Figure 2).

\section{rsFC differences between the SD group} and NS group

Compared with the NS group, in the SD group, the left CPL had increased rsFC with the right inferior frontal gyrus, right fusiform gyrus, right cingulate gyrus, right thalamus, and both sides of the PrC, and had decreased rsFC with the left middle frontal gyrus, left inferior frontal gyrus, and both sides of the superior frontal gyrus (Table 5, Figure 3).

Table 3 Altered resting-state functional connectivity areas of the left cerebellum posterior lobe after sleep deprivation

\begin{tabular}{|c|c|c|c|c|c|}
\hline \multirow[t]{2}{*}{ Brain } & \multirow[t]{2}{*}{$R / L$} & \multirow[t]{2}{*}{ Voxels } & \multicolumn{3}{|c|}{ MNI } \\
\hline & & & $\mathbf{x}$ & $\mathbf{Y}$ & $\mathbf{Z}$ \\
\hline \multicolumn{6}{|l|}{ Positive correlation } \\
\hline Cerebellum posterior lobe & L & 242 & -57 & -63 & -36 \\
\hline Cerebellum posterior lobe & $\mathrm{R}$ & 70 & 45 & -69 & -30 \\
\hline Cerebellum posterior lobe & L & 22 & -3 & -69 & -21 \\
\hline Posterior cingulate gyrus & L & 18 & 0 & -42 & 27 \\
\hline \multicolumn{6}{|l|}{ Negative correlation } \\
\hline Angular gyrus, precuneus & L & 20 & -33 & -75 & 30 \\
\hline Precentral gyrus, inferior frontal gyrus & L & 24 & -51 & 0 & 24 \\
\hline Postcentral gyrus, inferior parietal lobule & L & 52 & -51 & -33 & 39 \\
\hline Superior parietal lobule, inferior parietal lobule & L & 65 & -27 & -45 & 48 \\
\hline Middle frontal gyrus & $\mathrm{R}$ & 16 & 27 & 3 & 51 \\
\hline
\end{tabular}

Note: A significance level of $P<0.00$ I for individual voxels and a continuous voxel size $\geq 13$ were used to determine statistical significance, corrected for AlphaSim. Abbreviations: R, right; L, left; $\mathrm{MNI}$, Montreal Neurological Institute. 




Figure 2 Altered resting-state functional connectivity $(\mathrm{rsFC})$ areas of the cerebellum posterior lobe $(\mathrm{CPL})$ in the sleep deprivation group.

Notes: The rsFC areas of the left CPL were seen in the left precentral gyrus, left angular gyrus, left inferior frontal gyrus, left superior parietal lobule, left inferior parietal lobule, left postcentral gyrus, left precuneus, left posterior cingulate gyrus, right middle frontal gyrus, and bilaterally in the CPL (A), while the rsFC areas of the right CPL were seen in the left superior frontal gyrus, left middle frontal gyrus, left paracentral lobule, left superior temporal gyrus, left middle temporal gyrus, bilaterally in the cerebellum anterior lobe, and bilaterally in the CPL (B).

Compared with NS group, in the SD group, the right CPL had increased rsFC with the left superior frontal gyrus, and had decreased rsFC with the left precentral gyrus, left inferior frontal gyrus, right superior temporal gyrus, both sides of the superior frontal gyrus, and both sides of the middle frontal gyrus (Table 6, Figure 3).

\section{Discussion}

Anatomically, the "default-mode network" (DMN) spanned both sides of the IPL, posterior cingulate cortex, PrC, medial prefrontal cortex, retrosplenial cortex, and parts of the hippocampal formation and medial temporal lobe. ${ }^{36,37}$ The DMN was more active at rest than during stimulation and showed decreased activity during stimulation. ${ }^{36,38}$ Recent research has suggested that the DMN might be associated with the collection and evaluation of information, ${ }^{39}$ self-referential mental activity, ${ }^{40}$ extraction of episodic memory, ${ }^{41}$ emotion and anxiety, ${ }^{42,43}$ and mind wandering or daydreaming. ${ }^{44}$
To date, the focus of most research has been on delineating the regional effects of SD on DMN. ${ }^{45}$ In contrast, far less is known about the effect of SD on intrinsic low-frequency connectivity within the cerebellum and its anticorrelated network.

A previous study ${ }^{32}$ found that the rsFC network of the DMN contains a positive correlation network, consisting of task-related activations, and a negative correlation network, consisting of stimulation-related deactivations. The DMN was called the stimulation-negative network, and the other network was called the stimulation-positive network. ${ }^{32}$ The stimulation-positive network consists of regions routinely activated during goal-directed stimulation, including the dorsal-lateral and ventral prefrontal cortex, insula, and supplementary motor area.

In this study, left CPL showed the positive correlation regions with the task-positive network in NS group, and showed negative correlations with the regions of the DMN. This finding suggests that healthy volunteers have multiple

Table 4 Altered resting-state functional connectivity areas of the right cerebellum posterior lobe after sleep deprivation

\begin{tabular}{|c|c|c|c|c|c|}
\hline \multirow[t]{2}{*}{ Brain } & \multirow[t]{2}{*}{$\mathbf{R} / \mathbf{L}$} & \multirow[t]{2}{*}{ Voxels } & \multicolumn{3}{|c|}{ MNI } \\
\hline & & & $\mathbf{X}$ & $\mathbf{Y}$ & $\mathbf{Z}$ \\
\hline \multicolumn{6}{|l|}{ Positive correlation } \\
\hline Cerebellum anterior lobe & $\mathrm{L}$ & 23 & -15 & -51 & -27 \\
\hline Cerebellum posterior lobe & $\mathrm{L}$ & 38 & -24 & -39 & -48 \\
\hline Cerebellum anterior lobe, cerebellum posterior lobe & $\mathrm{R}$ & $\mathrm{I}, 027$ & 21 & -69 & -54 \\
\hline Cerebellum anterior lobe, cerebellum posterior lobe & $\mathrm{R}$ & 107 & 6 & -66 & -18 \\
\hline \multicolumn{6}{|l|}{ Negative correlation } \\
\hline Medial frontal gyrus, paracentral lobule, superior temporal gyrus & $\mathrm{L}$ & 28 & 0 & -21 & 72 \\
\hline Superior temporal gyrus, middle temporal gyrus & $\mathrm{L}$ & 13 & -57 & -12 & -3 \\
\hline
\end{tabular}

Note: A significance level of $P<0.00 \mathrm{I}$ for individual voxels and a continuous voxel size $\geq 13$ were used to determine statistical significance, corrected for AlphaSim. Abbreviations: R, right; L, left; MNI, Montreal Neurological Institute. 
Table 5 Altered resting-state functional connectivity regions of the left cerebellum posterior lobe after sleep deprivation versus normal sleep

\begin{tabular}{|c|c|c|c|c|c|}
\hline \multirow[t]{2}{*}{ Brain } & \multirow[t]{2}{*}{$\mathbf{R} / \mathbf{L}$} & \multirow[t]{2}{*}{ Voxels } & \multicolumn{3}{|c|}{ MNI } \\
\hline & & & $\mathbf{x}$ & $\mathbf{Y}$ & $\mathbf{Z}$ \\
\hline \multicolumn{6}{|l|}{ Increased areas } \\
\hline Inferior frontal gyrus & $\mathrm{R}$ & 32 & 54 & 33 & 12 \\
\hline Fusiform gyrus & $\mathrm{R}$ & 10 & 48 & -42 & -18 \\
\hline Precuneus & $\mathrm{L}$ & 11 & -18 & -75 & 36 \\
\hline Cingulate gyrus, precuneus & $\mathrm{R}$ & 14 & 15 & -39 & 36 \\
\hline Thalamus & $\mathrm{R}$ & 11 & 18 & -33 & 9 \\
\hline \multicolumn{6}{|l|}{ Reduced areas } \\
\hline Superior frontal gyrus & $\mathrm{R}$ & 26 & 18 & 48 & 33 \\
\hline Superior frontal gyrus, middle frontal gyrus, inferior frontal gyrus & $\mathrm{L}$ & 24 & -30 & 51 & 3 \\
\hline Middle frontal gyrus & L & 13 & -33 & 33 & 45 \\
\hline
\end{tabular}

Note: A significance level of $P<0.00 \mathrm{I}$ for individual voxels and a continuous voxel size $\geq 10$ were used to determine statistical significance, corrected for AlphaSim. Abbreviations: R, right; L, left; $\mathrm{MNI}$, Montreal Neurological Institute.

brain networks, and that these networks mutually confront each other and maintain a dynamic equilibrium. Furthermore, both the NS group and the SD group showed extensive rsFC between the left CPL and the regions of the DMN. However, no significant differences were found between the NS group and the SD group. The right CPL showed rsFC with the DMN in the NS group but not in the SD group, while the right CPL showed reduced rsFC with the regions of the DMN in the SD group compared with in the NS group. A previous study found that SD reduced the rsFC between DMN and the anti-correlation network during the rest and stimulation performance, ${ }^{45}$ which is associated with our finding that SD reduced the rsFC between DMN and the right CPL.

Significant differences in rsFC between the left CPL and right CPL were seen in the temporal lobe: the left CPL did not show any rsFC with the temporal lobe while the right $\mathrm{CPL}$ showed obvious rsFC with the temporal lobe, and the rsFC areas were more obvious in the NS group than in the SD group. We hypothesize that the right CPL is involved in accomplishing the sensation of acupuncture stimulation and SD reduces the rsFC regions between the right CPL and the temporal lobe.

The cerebellum anterior lobe receives the nociceptive afferent fibers of the spinal cord. Once the cerebellum anterior lobe is damaged, while there is inconspicuous cognitive and behavioral impairment. ${ }^{46}$ The CPL receives part of the fiber from the lateral and anterior spinothalamic tract at the same time, and reacts to noxious and non-noxious stimulation. In this study, we found that the right CPL showed rsFC with the cerebellum anterior lobe both in the NS group and in SD group, while the left CPL showed a lack of synchronism with

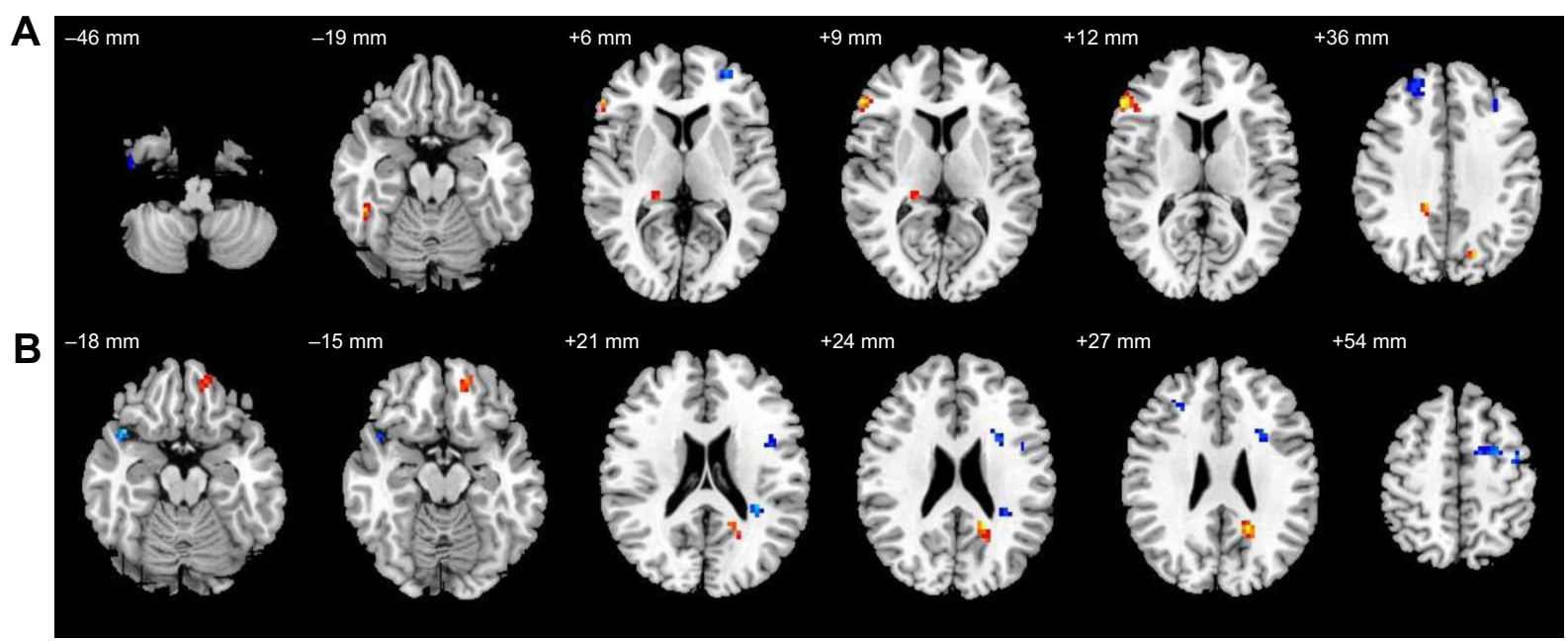

Figure 3 Altered resting-state functional connectivity $(\mathrm{rsFC})$ areas of the cerebellum posterior lobe (CPL) in the sleep deprivation group compared with in the normal sleep group.

Notes: The rsFC areas of the left CPL were seen in the left middle frontal gyrus, right fusiform temporal gyrus, right cingulate gyrus, right thalamus, bilaterally in the superior frontal gyrus, bilaterally in the inferior frontal gyrus, bilaterally in the precuneus (A), while the rsFC areas of the right CPL were seen in the left precentral gyrus, left inferior frontal gyrus, right superior temporal gyrus, bilaterally in the superior frontal gyrus, and middle frontal gyrus (B). 
Table 6 Altered resting-state functional connectivity regions of the right cerebellum posterior lobe after sleep deprivation versus normal sleep

\begin{tabular}{|c|c|c|c|c|c|}
\hline \multirow[t]{2}{*}{ Brain } & \multirow[t]{2}{*}{$R / L$} & \multirow[t]{2}{*}{ Voxels } & \multicolumn{3}{|c|}{ MNI } \\
\hline & & & $\mathbf{x}$ & $\mathbf{Y}$ & $\mathbf{Z}$ \\
\hline \multicolumn{6}{|l|}{ Increased areas } \\
\hline Superior frontal gyrus & L & 31 & -15 & 51 & -15 \\
\hline \multicolumn{6}{|l|}{ Reduced areas } \\
\hline Superior frontal gyrus, middle frontal gyrus & $\mathrm{R}$ & 29 & 12 & 3 & 69 \\
\hline Superior temporal gyrus & $\mathrm{R}$ & 13 & 42 & 15 & -18 \\
\hline Inferior frontal gyrus & $\mathrm{L}$ & II & -42 & 3 & 21 \\
\hline Middle frontal gyrus & L & 24 & -27 & 9 & 24 \\
\hline Middle frontal gyrus & $\mathrm{R}$ & 11 & 30 & 36 & 30 \\
\hline Superior frontal gyrus, middle frontal gyrus & L & 79 & -27 & -3 & 57 \\
\hline Precentral gyrus, middle frontal gyrus & L & 11 & -42 & -6 & 54 \\
\hline Precentral gyrus, middle frontal gyrus & L & 118 & -15 & -21 & 75 \\
\hline Superior frontal gyrus & $\mathrm{R}$ & 19 & 12 & 3 & 69 \\
\hline
\end{tabular}

Note: A significance level of $P<0.001$ for individual voxels and a continuous voxel size $\geq 10$ were used to determine statistical significance, corrected for AlphaSim. Abbreviations: R, right; L, left; MNI, Montreal Neurological Institute.

the cerebellum anterior lobe. Further, both sides of the CPL did not show any reduced rsFC with the cerebellum between the SD group and NS group. These findings suggest that the right $\mathrm{CPL}$ is more sensitive to receiving the nociceptive afferent fibers of the spinal cord than the left CPL, and the SD did not cause the abnormal rsFC between the CPL and cerebellum.

A previous study found that the regional homogeneity value of the parietal lobe was higher in an SD group than in an NS group. ${ }^{5}$ In support of this finding, in our study, we found that the left CPL had rsFC with the parietal lobe both in the NS group and in the SD group, there were more rsFC areas in the SD group than in the NS group, and the right CPL had rsFC with the parietal lobe only in the NS group. However, both left CPL and right CPL did not show any significant differences in $\mathrm{rsFC}$ with the parietal lobe between the SD group and NS group. These findings suggest that SD only reduced the rsFC areas in the parietal lobe and did not cause the abnormal rsFC between the CPL and the parietal lobe.

The CPL is widely used to adjust nerve function; complete functions such as cognition, language, and emotion; and initiate, plan, and coordinate movement. ${ }^{47,48}$ The frontal lobe is closely involved in sleep and cognitive function, such as working memory, attention, planning, behavior control, and information integration. ${ }^{49,50}$ The frontal lobe, an important part of the emotional central pathway, also plays an important role in mood regulation. ${ }^{51}$ Once the frontal lobe is impaired, emotional and social behavior are impaired. ${ }^{52}$ Anatomical studies have proved the existence of a connection between the frontal lobe and the cerebellum. ${ }^{53}$ In support of this finding, in our study, we found that both the left CPL and right CPL showed rsFC with the frontal lobe, and there were more rsFC areas in the frontal lobe in the NS group than in SD group. Our finding suggests that SD reduced the number of rsFC areas between both sides of the CPL and the frontal lobe. The cerebellum, to form a feed-forward loop through the thalamus and to form a feedback loop through the pons, interconnects a network with extensive cortical and subcortical areas. ${ }^{16}$ These anatomic connections of extensive cortical and subcortical areas support the role of the cerebellum in cognitive and emotional processing. ${ }^{16} \mathrm{~A}$ large body of empirical research in patients with cerebellar damage has demonstrated that the region is related to cognitive and emotional regulation. ${ }^{16,17} \mathrm{~A}$ previous study found that SD caused abnormal changes in the whole network by connecting multiple brain regions, which had a significant influence on advanced functions, such as cognition and emotion, by mainly damaging the frontal lobe and cerebellum. ${ }^{22,37}$ In support of this finding, in our study, we found that both the left CPL and right CPL showed reduced rsFC with the frontal lobe in the SD group compared with the NS group, and the reduction was more significant in the right CPL than in the left CPL. Our findings suggest that SD causes rsFC impairment between both sides of the CPL and the frontal lobe, and the right CPL showed more significant rsFC impairment than the left CPL. Abnormal rsFC may be one of the most important reasons why emotional and cognitive function changes.

\section{Conclusion}

The bilateral CPL are possibly involved in acupuncture stimulation in different manners: the left CPL showed lack 
of synchronism with the temporal lobe and the cerebellum anterior lobe in both the NS group and SD group, while the right CPL showed lack of synchronism with the parietal lobe. SD caused rsFC impairment between the right CPL and the frontal lobe, temporal lobe and DMN, and rsFC impairment between the left CPL and frontal lobe. Our findings provide insights into the pathophysiological mechanism of SD and may be helpful for understanding the cerebellum.

\section{Acknowledgments}

This work was supported by Jiangxi Provincial Department of Science and Technology Support Program (grant numbers 20121BBG70056, 20132BBG70061, and 20141BBG70026), and Jiangxi Provincial Department of Natural Science Foundation Project (grant number 20132BAB205100).

\section{Disclosure}

The authors declare no conflicts of interest in this work.

\section{References}

1. Drummond SP, Brown GG. The effects of total sleep deprivation on cerebral responses to cognitive performance. Neuropsychopharmacology. 2001;25(5 Suppl):68-73.

2. Jackson ML, Huqhes ME, Croft RJ, et al. The effect of sleep deprivation on BOLD activity elicited by a divided attention task. Brain Imaging Behav. 2011;5(2):97-108.

3. Luber B, Stanford AD, Bulow P, et al. Remediation of sleep-deprivationinduced working memory impairment with fMRI-guided transcranial magnetic stimulation. Cereb Cortex. 2008;18(9):2077-2085.

4. Nilsson JP, Söderström M, Karlsson AU, et al. Less effective executive functioning after one night's sleep deprivation. $J$ Sleep Res. 2005;14(1):1-6.

5. Dai XJ, Gong HH, Wang YX, et al. Gender differences in brain regional homogeneity of healthy subjects after normal sleep and after sleep deprivation: a resting-state fMRI study. Sleep Med. 2012;13(6): $720-727$.

6. Ohayon MM, Smolensky MH, Roth T. Consequences of shiftworking on sleep duration, sleepiness, and sleep attacks. Chronobiol Int. 2010;27(3):575-589.

7. Tsigos C, Chrousos GP. Hypothalamic-pituitary-adrenal axis, neuroendocrine factors and stress. J Psychosom Res. 2002;53(4):865-871.

8. Walker MP, Stickgold R. Sleep, memory, and plasticity. Annu Rev Psychol. 2006;57:139-166.

9. Ivry RB, Keele SW. Timing functions of the cerebellum. J Cogn Neurosci. 1989;1(2):136-152.

10. Fiez JA, Petersen SE, Cheney MK, Raichle ME. Impaired non-motor learning and error detection associated with cerebellar damage. A single case study. Brain. 1992;115(1):155-178.

11. Courchesne E, Townsend J, Akshoomoff NA, et al. Impairment in shifting attention in autistic and cerebellar patients. Behav Neurosci. 1994;108(5):848-865.

12. Allen G, Buxton RB, Wong EC, Courchesne E. Attentional activation of the cerebellum independent of motor involvement. Science. 1997;275(5308):1940-1943.

13. Le TH, Pardo JV, Hu X. 4 T-fMRI study of nonspatial shifting of selective attention: cerebellar and parietal contributions. J Neurophysiol. 1998; 79(3): 1535-1548.

14. Schmahmann JD, Sherman JC. The cerebellar cognitive affective syndrome. Brain. 1998;121(4):561-579.
15. Parvizi J, Anderson SW, Martin CO, Damasio H, Damasio AR. Pathological laughter and crying: a link to the cerebellum. Brain. 2001;124 (Pt 9):1708-1719.

16. Dai XJ, Peng DC, Gong HH, et al. Altered intrinsic regional brain spontaneous activity and subjective sleep quality in patients with chronic primary insomnia: a resting-state fMRI study. Neuropsychiatr Dis Treat. 2014;10:2163-2175.

17. Allen G, Courchesne E. Differential effects of developmental cerebellar abnormality on cognitive and motor functions in the cerebellum: an fMRI study of autism. Am J Psychiatry. 2003;160(2):262-273.

18. Liu ZF, Xu C, Xu Y, et al. Decreased regional homogeneity in insula and cerebellum: a resting-state fMRI study in patients with major depression and subjects at high risk for major depression. Psychiatry Res. 2010;182(3):211-215.

19. Soares JC, Mann JJ. The anatomy of mood disorders - review of structural neuroimaging studies. Biol Psychiatry. 1997;41(1):86-106.

20. Peng DC, Dai XJ, Gong HH, Li HJ, Nie X, Zhang W. Altered intrinsic regional brain activity in male patients with severe obstructive sleep apnea: a resting-state functional magnetic resonance imaging study. Neuropsychiatr Dis Treat. 2014;10:1819-1826.

21. Carotenuto M, Gallai B, Parisi L, Roccella M, Esposito M. Acupressure therapy for insomnia in adolescents: a polysomnographic study. Neuropsychiatr Dis Treat. 2013;9:157-162.

22. Dai XJ, Min YJ, Gong HH, et al. [Evaluation of the post-effect of acupuncture at Sanyinjiao (SP 6) under sleep deprivation by resting-state amplitude of low-frequency fluctuation: a fMRI study.] Zhongguo Zhen Jiu. 2012;32(1):47-52. Chinese.

23. Wu MT, Hsieh JC, Xiong J, et al. Central nervous pathway for acupuncture stimulation: localization of processing with functional MR imaging of the brain - preliminary experience. Radiology. 1999;212(1):133-141.

24. Kong J, Ma L, Gollub RL, et al. A pilot study of functional magnetic resonance imaging of the brain during manual and electroacupuncture stimulation of acupuncture point (LI-4 Hegu) in normal subjects reveals differential brain activation between methods. $J$ Altern Complement Med. 2002;8(4):411-419.

25. Gao L, Zhang M, Gong H, et al. Differential activation patterns of fMRI in sleep-deprived brain: restoring effects of acupuncture. Evid Based Complement Alternat Med. 2014;(2014):Article ID 465760.

26. Yoo SS, Teh EK, Blinder RA, Jolesz FA. Modulation of cerebellar activities by acupuncture stimulation: evidence from fMRI study. Neuroimage. 2004;22(2):932-940.

27. Dai XJ, Liu CL, Gong HH, et al. Long-term total sleep deprivation decreases the default spontaneous activity and connectivity pattern in healthy male subjects: a resting-state fMRI study. Neuropsychiatr Dis Treat. 2015;11:761-772.

28. Li HJ, Dai XJ, Gong HH, et al. Aberrant spontaneous low-frequency brain activity in male patients with severe obstructive sleep apnea revealed by resting-state functional MRI. Neuropsychiatr Dis Treat. 2015;11:207-214.

29. Horwitz B, Warner B, Fitzer J, Tagamets MA, Husain FT, Long TW. Investigating the neural basis for functional and effective connectivity. Application to fMRI. Philos Trans R Soc Lond B Biol Sci. 2005; 360(1457):1093-1108.

30. Satterthwaite TD, Elliott MA, Gerraty RT, et al. An improved framework for confound regression and filtering for control of motion artifact in the preprocessing of resting-state functional connectivity data. Neuroimage. 2013;64:240-256.

31. Yan CG, Cheung B, Kelly C, et al. A comprehensive assessment of regional variation in the impact of head micromovements on functional connectomics. Neuroimage. 2013;76:183-201.

32. Fox MD, Snyder AZ, Vincent JL, Corbetta M, Van Essen DC, Raichle ME. The human brain is intrinsically organized into dynamic, anticorrelated functional networks. Proc Natl Acad Sci U S A. 2005;102(27): 9673-9678.

33. Saad ZS, Gotts SJ, Murphy K, et al. Trouble at rest: how correlation patterns and group differences become distorted after global signal regression. Brain Connect. 2012;2(1):25-32. 
34. Gao L, Bai L, Zhang Y, et al. Frequency-dependent changes of local resting oscillations in sleep-deprived brain. PLoS One. 2015;10(3): e0120323.

35. Dai XJ, Liu BX, Min YJ, et al. Different cerebellar responding to acupuncture at SP6 under different sleep states: an fMRI study. J Sleep Disord Treat Care. 2013;2:2.

36. Buckner RL, Andrews-Hanna JR, Schacter DL. The brain's default network: anatomy, function, and relevance to disease. Ann N Y Acad Sci. 2008;1124:1-38.

37. Fox MD, Raichle ME. Spontaneous fluctuations in brain activity observed with functional magnetic resonance imaging. Nat Rev Neurosci. 2007;8(9):700-711.

38. Raichle ME, MacLeod AM, Snyder AZ, Powers WJ, Gusnard DA, Shulman GL. A default mode of brain function. Proc Natl Acad Sci US A. 2001;98(2):676-682.

39. Gusnard DA, Raichle ME, Raichle ME. Searching for a baseline: functional imaging and the resting human brain. Nat Rev Neurosci. 2001; 2(10):685-694.

40. Gusnard DA, Akbudak E, Shulman GL, Raichle ME. Medial prefrontal cortex and self-referential mental activity: relation to a default mode of brain function. Proc Natl Acad Sci US A. 2001;98(7):4259-4264.

41. Cabeza R, Dolcos F, Graham R, Nyberg L. Similarities and differences in the neural correlates of episodic memory retrieval and working memory. Neuroimage. 2002;16(2):317-330.

42. Simpson JR Jr, Drevets WC, Snyder AZ, Gusnard DA, Raichle ME. Emotion-induced changes in human medial prefrontal cortex: II. During anticipatory anxiety. Proc Natl Acad Sci U S A. 2001;98(2): 688-693.

43. Simpson JR Jr, Snyder AZ, Gusnard DA, Raichle ME. Emotion-induced changes in human medial prefrontal cortex: I. During cognitive task performance. Proc Natl Acad Sci U S A. 2001;98(2):683-687.
44. Mason MF, Norton MI, Van HornJD, WegnerDM, Grafton ST, MacraeCN. Wandering minds: the default network and stimulus-independent thought. Science. 2007;315(5810):393-395.

45. De Havas JA, Parimal S, Song CS, Chee MW. Sleep deprivation reduces default mode network connectivity and anti-correlation during rest and task performance. Neuroimage. 2012;59(2):1745-1751.

46. Paulus KS, Maqnano I, Conti M, et al. Pure post-stroke cerebellar cognitive affective syndrome: a case report. Neurol Sci. 2004;25(4): 220-224.

47. Barrios M, Guàrdia J. [Relation of the cerebellum with cognitive function: neuroanatomical, clinical and neuroimaging evidence.] Rev Neurol. 2001;33(6):582-591. Spanish.

48. Desmond JE, Marvel CL. Cognition: cerebellum role. In: Squire LR, editor. Encyclopedia of Neuroscience. Vol 2. Oxford: Academic Press; 2009:1079-1085.

49. Goel V, Dolan RJ. Differential involvement of left prefrontal cortex in inductive and deductive reasoning. Cognition. 2004;93(3):B109-B121.

50. Christoff K, Prabhakaran V, Dorfman J, et al. Rostrolateral prefrontal cortex involvement in relational integration during reasoning. Neuroimage. 2001;14(5):1136-1149.

51. Phillips ML, Drevets WC, Rauch SL, Lane R. Neurobiology of emotion perception I: The neural basis of normal emotion perception. Biol Psychiatry. 2003;54(5):504-514.

52. Davidson RJ. Anxiety and affective style: role of prefrontal cortex and amygdala. Biol Psychiatry. 2002;51(1):68-80.

53. Middleton FA, Strick PL. Cerebellar projections to the prefrontal cortex of the primate. J Neurosci. 2001;21(2):700-712.
Neuropsychiatric Disease and Treatment

\section{Publish your work in this journal}

Neuropsychiatric Disease and Treatment is an international, peerreviewed journal of clinical therapeutics and pharmacology focusing on concise rapid reporting of clinical or pre-clinical studies on a range of neuropsychiatric and neurological disorders. This journal is indexed on PubMed Central, the 'PsycINFO' database and CAS,

\section{Dovepress}

and is the official journal of The International Neuropsychiatric Association (INA). The manuscript management system is completely online and includes a very quick and fair peer-review system, which is all easy to use. Visit http://www.dovepress.com/testimonials.php to read real quotes from published authors. 